\title{
MEG AS A MEDICAL DIAGNOSTIC TOOL IN THE GREEK POPULATION
}

\author{
Photios Anninos, Adam Adamopoulos, Athanasia Kotini
}

Lab of Medical Physics, Medical School, Democritus University of Thrace, Alexandroupolis, Greece

\begin{abstract}
Summary: Magnetoencephalography (MEG) is the recording of the magnetic field produced by the flowing of ions in the brain. This article reports our experience in the application of MEG in patients and healthy volunteers in the Greek population. We provide a brief description of our research work. The MEG data were recorded in a magnetically shielded room with a whole-head 122 channel or an one-channel biomagnetometer. Our results lead us to believe that the MEG is an important research field which is evolving quickly with a number of interesting findings with respect to normal and abnormal functions of the human brain. It could provide clinical practice with an easy to perform non invasive method, which could be adjunct to conventional methods for the evaluation of brain disorders.
\end{abstract}

Keywords: MEG; SQUID; Epilepsy; Febrile seizures; Parkinson; Multiple sclerosis; Alzheimer

\section{Introduction}

Magnetoencephalography (MEG) is a noninvasive imaging technique, applicable to the human brain with a temporal resolution of approximately $\sim 1 \mathrm{~ms}$. In MEG, weak magnetic fields generated by electric currents in the brain are measured using Superconducting Quantum Interference Devices (SQUIDs) positioned on the skull. Time varying electric currents, in wires or brain cells, produce time-varying magnetic fields. Even though transmembrane, intracellular and extracellular neuronal currents each produce a surrounding magnetic flux, the neuromagnetic field recordable outside of the head is a selective reflection of intracellular currents flowing in the apical dendrites of pyramidal cells oriented parallel to the skull surface. This is a result of the biophysical properties of the neuronal currents and the volume conduction properties of the head. The output of each channel of the biomagnetometer is a time varying voltage waveform that reflects local changes in magnetic flux as a function of time. MEG is one of the non-invasive functional brain imaging techniques. Analysis of brain function by fMRI and PET is based on the change of cerebral blood flow induced by neural activity and that of EEG and MEG on the electric potential and magnetic changes induced by neural activity, respectively. Consequently, EEG and MEG feature higher temporal resolutions than PET and fMRI in measurements of brain activity.

In addition external transcranial magnetic stimulation (TMS) was applied to patients with CNS disorders with proper characteristics (magnetic field in the order of $\mathrm{pT}$ and frequency the alpha rhythm of the patient $(8-13 \mathrm{~Hz})$. The MEG recordings after the application of TMS shown a rapid attenuation of the abnormal MEG activity followed by an increase of the low frequency components and the alpha rhythm of the patients. Further signal analysis indicated that the application of the TMS strongly influenced the underlying brain dynamics with beneficial effects on the clinical status of the patients with CNS disorders.

This article reports our research work dealing with MEG as a diagnostic tool in medicine and its use in conjunction with other stimulation and theoretical methods for the evaluation of various diseases.

\section{Methods}

We employed the one-channel MEG system in 1985 and the 122-channel MEG system in 1999 from Greek Research programs. The 122-channel system provides a helmet shaped detector array covering the entire head allowing simultaneous recording of magnetic fields over the whole cortex and have a lot of advantages over the one-channel system.

\section{A. 122-channel MEG system}

The MEG recordings were carried out in a magnetically shielded room with a whole head 122-channel biomagnetometer (model: Neuromag-122, Helsinki, Finland) (Figure 1). During the recordings the subject was sitting in a chair with his/her head covered by the helmet-shaped dewar. The MEG sampling frequency was $256 \mathrm{~Hz}$ and the associated Nyquist frequency was $128 \mathrm{~Hz}$, which was well above of the constituent frequency components of interest in our MEG recordings, thus avoiding aliasing artefacts. The time taken for each MEG recording was $3 \mathrm{~min}$. For each MEG spike, we calculated the single equivalent current dipole (ECD) source at the spike peak. It was suitable for our measurements regarding localization of epileptic foci. We defined acceptable 


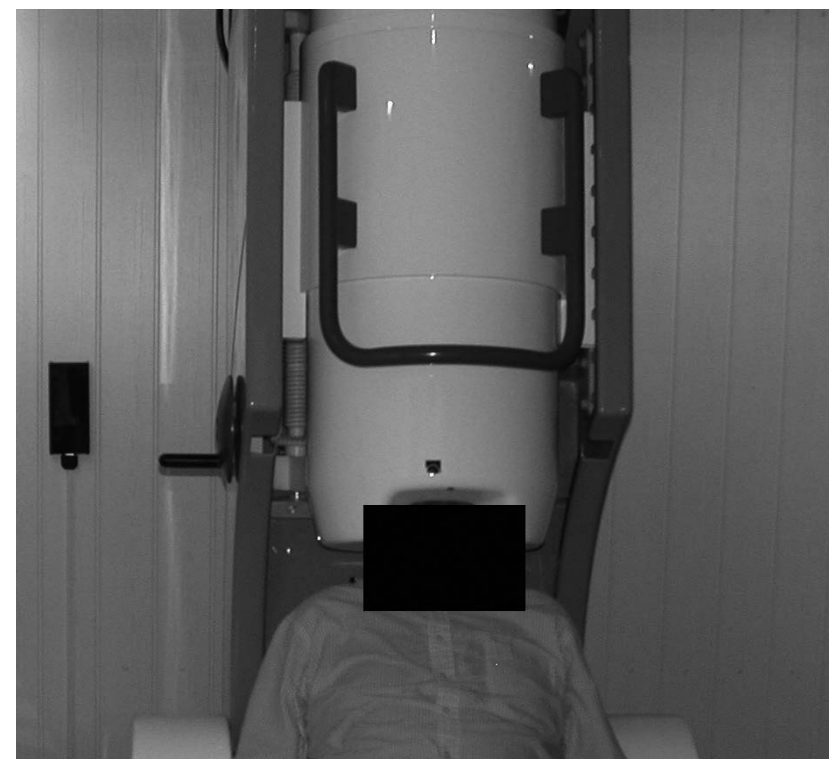

Fig. 1: The 122-channel MEG system with the patient during the measurements.
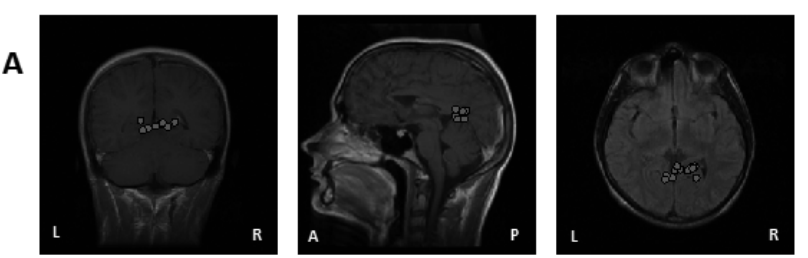

B
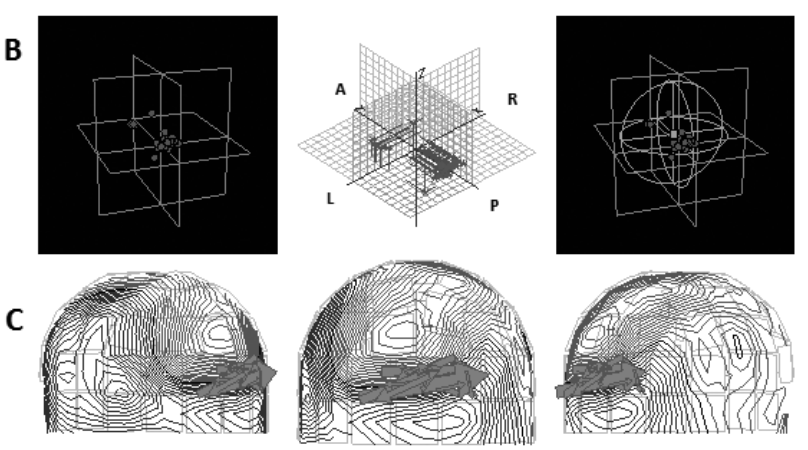

Posterior

D

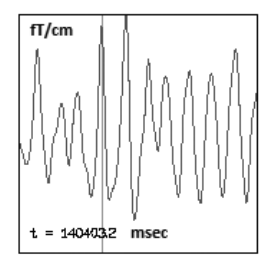

Fig. 2: A) Coronal, sagittal and axial MRI images for one patient with the superposition of the active ECDs indicated by the red dots. (L: left, R: right, A: anterior, P: posterior) B) The whole number of dipoles in the $\mathrm{x}, \mathrm{y}, \mathrm{z}$ axes. C) The scalp isocontour filed distribution and the ECDs indicated by the green arrows. D) The MEG raw data for a time duration where the ECDs were specified. The $\mathrm{x}$ axis is the time in msec and the $y$ axis is the magnetic field in $\mathrm{fT} / \mathrm{cm}$ $(\mathrm{fT}=$ femto Tesla $)$.
ECDs as those with a goodness-of-fit to the model of $>70 \%$ and with ECD strength between 100 and 400 nAm (nano Ampere metre). Three fiducial points were defined on each patient's head surface which were clear anatomic landmarks (the 2 preauricular points and the nasion). These 3 points define the coordinate system that includes the brain and the position of the magnetometers relative to it. The line between the preauricular points defines the $\mathrm{x}$-axis of the coordinate system, with the positive direction being to the right. The line between the nasion and the mid-point of the x-axis and perpendicular to it, defines the y-axis and the line perpendicular to the $x-y$ plane, passing through the intersection of the $x$ and $y$-axes, defines the $z$-axis of the coordinate system. The positive $y$-axis passed through the nasion and the $\mathrm{z}$-axis pointed upwards. The locations of the ECDs were estimated and projected onto the structural images of the brain (MRI), that displays the activated brain regions (Figure 2).

\section{B. One-channel MEG system}

Biomagnetic measurements were performed using a one channel second order gradiometer SQUID model 601 of the Biomagnetic Technologies Inc. (Figure 3), which was located in an electrically shielded room. The MEG recordings were performed after positioning the SQUID sensor $3 \mathrm{~mm}$ above the scalp of the patient, with the use of an optic positioning system, which was based on the International 10-20 Electrode Placement System. This system uses any one of the standard EEG recording positions as its origin. We used the P3, P4, T3, T4, F3 and F4 recording positions. The reference system was devised to retrieve maximal information from a specified area of the skull given that the gradiometer coil is theoretically equally sensitive to all magnetic flux lines perpendicular to a circular area of the brain. In our case, this circle has been an effective diameter of $2.36 \mathrm{~cm}$, i.e. the diameter of the SQUID sensor coil. A rectangular 32-point matrix was used around the origin ( 4 rows $\times 8$ columns, equidistantly spaced in a $4.5 \mathrm{~cm} \times 10.5 \mathrm{~cm}$ rectangle) for positioning of the SQUID. The MEG was recorded from each cerebral hemisphere at each of the 32 matrix points on the scalp for 32 consecutive epochs. Each epoch was of $1 \mathrm{sec}$ duration and was digitized with a sampling frequency of $256 \mathrm{~Hz}$. The MEG signal was band-pass filtered with cut-off frequencies of 0.1 and $60 \mathrm{~Hz}$. The MEG recordings were digitized using a 12 bit precision analog to digital converter with a sampling frequency of $256 \mathrm{~Hz}$, and were stored in a PC peripheral memory for off-line Fourier statistical analysis. With this technique we obtained two dimensional isocontour spectral amplitude maps (ISO-SA maps) (Figure 4). These maps were useful in obtaining clearly defined areas of high spectral density in the $2-7 \mathrm{~Hz}$ band frequencies. In addition they were helpful in providing clear identification of the coordinates of the point on the scalp where the MEG power spectrum has its maximal power as well as its maximal magnetic field intensity. We investigated the $2-7 \mathrm{~Hz}$ frequency band because we were interested in 


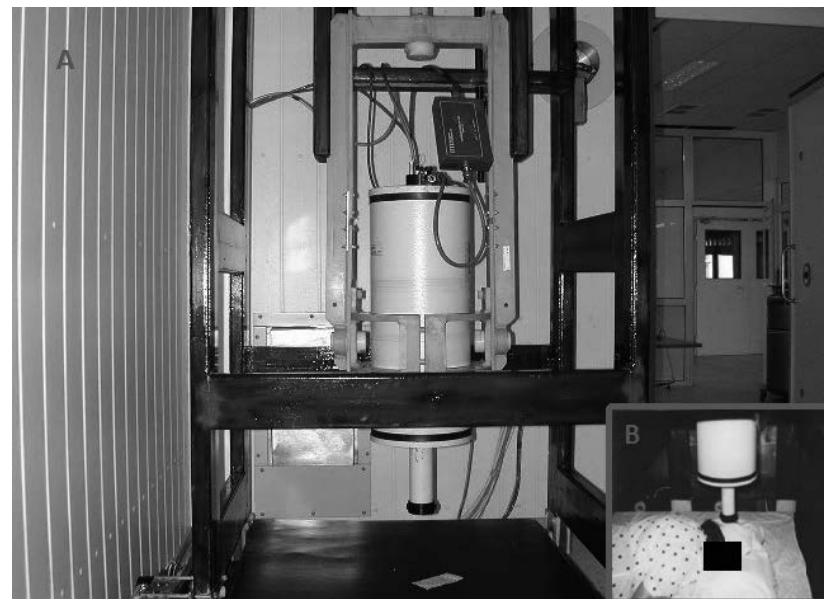

Fig. 3: A) The one-channel MEG system. B) The patient during the measurement.

the behavior of delta and theta rhythms. Figure 4 shows the left temporal lobe of an epileptic patient before and after the application of TMS. We observe a significant reduction of the magnetic field in the spectral amplitude maps after the application of TMS.

\section{Results}

\section{A. 122-channel MEG system}

\section{Gustatory states}

Gemousakakis et al. (1) evaluated MEG recordings for healthy female volunteers, in five different gustatory states: normal, sweet, bitter, sour and salty in all studied states. There was a higher number of low frequencies and a lower number of high frequencies with increasing age. Statistically significant differences were found in the normal and sweet states for the frequencies of $2 \mathrm{~Hz}$ and $7 \mathrm{~Hz}$ and in the salty taste in the frequency of $7 \mathrm{~Hz}$. They also intra-compared the

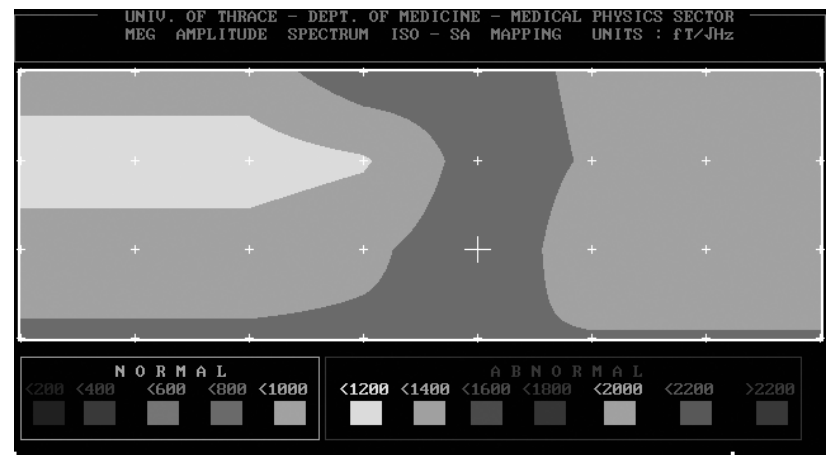

five states in group A and the five states in group B for the $2 \mathrm{~Hz}$ and $7 \mathrm{~Hz}$ frequencies. The results were not statistically significant.

Gemousakakis et al. (2) evaluated MEG recordings in five different states: normal condition, sweet, bitter, sour, and salty taste. The results showed that, in the normal condition, as well as in the sweet and the bitter taste, the male volunteers exhibited a higher count of low-frequency than high-frequency channels compared to the female ones; in the case of the sour taste, there was no clear differentiation between the genders; for the salty taste, the female volunteers exhibited a higher number of low-frequency channels whereas there was no clear differentiation in the number of high frequencies between the gender.

Anninos et al. (3) investigated the localization of current sources for spontaneous MEG data in the frequency domain. MEGs were evaluated in three different states: physiological, sweet and salty. Low frequencies can be seen in the maps obtained with the sweet taste, whereas in the physiological and salty taste, the maps showed higher frequencies in the majority of channels.

\section{Epilepsy}

Antoniou et al. (4) revealed quantitative differences in the MEG received from patients with Idiopathic Generalized Epilepsy (IGE) and from healthy volunteers. The analysis demonstrated the existence of spatially diffused low dimensionality in the MEG signals of patients with IGE.

Kotini et al. (5) investigated the electromagnetic sources of epileptic activity in two patients with juvenile myoclonus epilepsy (JME). The MRI examinations for both patients did not disclose any focal lesions or areas of abnormal signal intensity or enhancement by contrast media. MEG was recorded 5 years after the disease onset for the first patient and 11 years for the second patient. For the first patient dipolar sources of MEG paroxysmal activity were localized at the vermis and extending up to the occipital region, whereas,

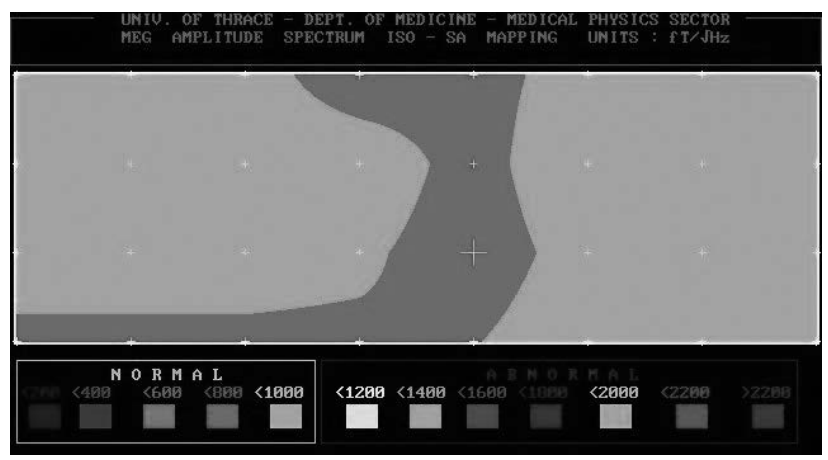

Fig. 4: The ISO-SA map of the left temporal area of an epileptic patient A) before and B) after the application of TMS. The large symbol "+" represents the reference point for the left hemisphere for easy identification of the coordinates of the functional focal points. The white small symbols "+" represent the 32 measured points with respect to the reference points. The maximal total average emitted power in the $2-7 \mathrm{~Hz}$ frequency band was $>1000 \mathrm{fT} / \sqrt{ } \mathrm{Hz}$ before TMS and became $<1000 \mathrm{fT} / \sqrt{ } \mathrm{Hz}$ afterwards. 
for the second patient dipolar sources of MEG paroxysmal activity were localized at the cerebellar area (vermis and hemisphere).

Anninos et al. (6) assessed any cerebral dysfunction in young children, who experienced febrile seizures, by means of MEG. ECDs were calculated for epileptic spikes on MEG recordings according to the ECD. Of 15 children, 8 showed ECDs located at the left-temporal, right-temporal, occipital, and frontal lobe, as active regions responsible for febrile seizures. They assumed that the interictal epileptiform discharges were a consequence of febrile seizures.

Kotini et al. (7) investigated the localization of current sources from spontaneous MEG data recorded from epileptic patients randomly selected, with different types of epilepsy. For each MEG spike, they calculated the ECD sources at the initial spike peaks with a spherical model. For each patient there was an increase of the frequency range after the ECD in compared to the one before the ECD. This was present in the whole study group due to epileptic discharge which was statistically significant. There was also a statistically significant difference in the increase of the frequency range in four patients with pathologic MRI, in five patients with normal MRI, in five patients with a high incidence of seizures and in four patients with onset age $<10$ years.

\section{Alzheimer Disease}

Abatzoglou et al. (8) obtained MEG from Alzheimer Disease (AD) patients and analyzed them using Fourier Transform in order to get the frequency distribution of MEG values. From this evaluation it was concluded that in patients with $\mathrm{AD}$ the dominant frequencies were significantly lower compared to normal individuals.

\section{Transcranial Magnetic Stimulation (TMS)}

\section{TMS and CNS Disorders}

Anninos et al. (9) applied external transcranial magnetic stimulation (TMS) to patients with proper characteristics which were obtained with MEG recordings prior to TMS. The MEG recordings after the application of TMS showed a rapid attenuation of the high abnormal activity followed by an increase in the number of the low frequency components toward the patients alpha-rhythm. The possible mechanisms of the effects of TMS on the pineal gland in the brain were presented.

\section{TMS and Parkinson Disease}

Anninos et al. (10) applied TMS in the order of pico Tesla on the patients with proper field characteristics which were obtained prior to TMS. The patients responded to the TMS with a feeling of relaxation and partial or complete disappearance of tremor, muscular ache and levodopa induced dyskinesias as well as rapid reversed visuospatial impairment, which were followed by a corresponding improvement and normalization of the MEG.

\section{Parkinson Disease}

Kotini et al. (11) investigated the MEG recordings from PD patients and healthy volunteers in the frequency domain. Prominent low frequencies can be seen in the spectrum obtained from the PD patients. MRI did not disclose specific findings in any case.

\section{Malignant CNS lesions}

Antoniou et al. (12) presented recordings from 2 patients diagnosed with malignant CNS lesions. By applying the Grassberger-Procaccia method of phase space reconstruction to the MEG of patients with malignant CNS lesions they found clear evidence linking the embedding dimension $\mathrm{m}$ in which they had the onset of saturation with the progress of the treatment.

Antoniou et al. (13) investigated the MEG from patients with malignant CNS lesions and from healthy volunteers. Evidence linking MEG signal characteristics (existence of low dimensionality chaotic dynamics) with the existence of the tumour was found from this analysis.

\section{B. One-channel MEG system}

\section{Alzheimer Disease}

Abatzoglou et al. (14) applied non-linear analysis on MEG signals of Alzheimer Disease (AD) patients in order to investigate the underlying complexity of the brain dynamics. Some recorded points were found with high amplitudes and low frequencies in magnetic activity. By applying nonlinear analysis in these records, low values in the correlation dimension $\mathrm{D}$ of the reconstructed phase space were found.

\section{Multiple sclerosis}

Kotini et al. (15) investigated multiple sclerosis (MS) patients. Some of the recorded points were observed to exhibit abnormal rhythmic activity, characterized by lower amplitudes and frequencies compared with controls. Using the MEG brain activity they were able to obtain a mapping technique characterized by the spectral amplitude of scalp distribution.

Kotini et al. (16) investigated if there was any nonlinearity in the MEG recordings of patients with MS in comparison with controls in order to find out the differences in the mechanisms underlying their brain waves. Chaotic activity of MS patients was lower than in the normal brain.

Transcranial Magnetic Stimulation (TMS)

\section{TMS and Arachnoid Cyst}

Anninos et al. (17) reported a 34-year-old male diagnosed of an intracranial arachnoid cyst arising in the left-sided temporal-parietal area that underwent evaluation by means of 
MEG. Biomagnetic waveform recordings were obtained from the target area and Fourier analysis of these measurements was carried out. External TMS in the order of pico Tesla was applied with proper field characteristics which were obtained prior to the application and the emitted MEG activity was recorded again. The cortical area adjacent to the borders of the arachnoid cyst emitted biomagnetic waveforms with high values. The application of TMS resulted in a rapid attenuation of the high MEG activity in the target area.

\section{TMS and Epstein Barr}

Anninos et al. (18) reported a 2-years-old male infant with a history of Epstein-Barr virus encephalitis, treated with foscarnet and steroids, because he developed mutism, ataxia and loss of the ability to eat, walk and talk. Brain imaging revealed loss of white matter around ventricles and progressive global brain atrophy, findings consistent with encephalopathy. There was an improvement of the clinical findings after the application of TMS with proper field characteristics. This case illustrated the possibility of therapeutic applications of TMS with proper field characteristics to normalize pathologically decreased levels of brain cortex activity.

\section{TMS and Alopecia universalis}

Anninos et al. (19) used MEG measurements and external magnetic field in the differential diagnosis and management of gynaecological and neurological entities. Exogenous TMS and MEG findings had been applied in the treatment of an alopecia universalis case. Important hair regrowth appeared progressively from the 2 nd week till the 15 th month of the therapeutic protocol. The indubitable superiority (comparatively to the control group) was noted.

\section{TMS and Epilepsy}

Anninos et al. (20) investigated the influence of external TMS in epileptic patients using MEG measurements and non-linear analytic techniques. In order to investigate the existence of any alteration in the complexity underlying the neural dynamics characterizing the pathologic brain before and after the TMS, a chaotic analysis approach was applied for the estimation of the dimensional analysis of the existing strange attractors. The application of TMS resulted in rapid attenuation of the MEG activity of epileptic patients. The obtained results of the dimensionality calculation provided a shift from lower to higher dimensional values, which is an indication that the system is chaotic.

Anninos et al. (21) presented three randomly selected epileptic patients in which application of external TMS of low intensities and frequencies produced a substantial attenuation of their abnormal brain activity. Furthermore, they presented a statistical analysis of 50 randomly selected epileptic patients who underwent TMS for the treatment of their seizures and they found that the anticonvulsant response to TMS was statistically significant.

Anninos et al. (22) measured the MEG from a patient with history of tonic-clonic seizures over 27 years who, despite medical treatment, experienced 8-10 convulsions daily. Subsequently, they energized an electronic device, which emitted magnetic fields with proper frequencies and intensities, and returned these back to the subject's brain. After magnetic stimulation with this electronic device, the seizures stopped.

Anninos et al. (23) developed a mapping technique using the MEG brain activity from epileptic patients. In addition by utilizing the characteristics of the Fourier power spectrum from the recorded MEG data they were also able to energize a magnetic stimulator which emits back to the patient's brain TMS with proper frequencies and intensities. Furthermore chaotic analysis approach was applied for the estimation of the dimensional analysis of the existing strange attractors. The obtained results of the dimensionality estimation furnish strong evidence of a shift of the correlation dimension to higher values after the application of TMS.

\section{TMS and Parkinson Disease}

Anninos et al. (24) obtained MEG recordings from patients suffering from Parkinson's disease (PD). External TMS was applied and the brain magnetic activity was recorded again. The application of TMS resulted in rapid attenuation of the MEG activity of PD patients. Furthermore, chaotic dynamic methods were used, in order to estimate the correlation dimension $\mathrm{D}$ of the reconstructed phase spaces. The estimated values of $D$, in conjunction with the results derived from the other data analysis methods, strongly supported the existence of low dimension chaotic structures in the dynamics of cortical activity of PD patients.

\section{TMS and Multiple Sclerosis}

Anninos et al. (25) used MEG recordings from MS patients and applied TMS back to the MS patients brain with proper frequencies and intensities. Furthermore they applied non-linear analysis in order to investigate if there was any alteration in the complexity underlying the dynamics, which characterized the brain of MS patients before and after TMS. The obtained results of the dimensionality estimation furnished a strong evidence of a shift of the correlation dimension to a higher value after the application of TMS.

\section{TMS and Meniere Syndrome}

Anninos et al. (26) investigated Meniere's syndrome. Using MEG they obtained the magnetic power spectrum distribution and utilizing its characteristics, they applied TMS back to the patient's brain. The brain activity was recorded and analyzed again, and by applying non-linear analysis they were able to investigate the brain's complexity. The results of the dimensionality estimation furnished a shift of the correlation dimension to higher values.

\section{Theoretical model and Epilepsy}

Kotini et al. (27) compared a theoretical neural net model with MEG data from epileptic patients and normal individ- 
uals. Using the method of $\mathrm{x}^{2}$-fitting it was found that the MEG amplitudes in epileptic patients and normal subjects had Poisson and Gauss distributions respectively. The Poisson connectivity derived from the theoretical neural model represented the state of epilepsy, whereas the Gauss connectivity represented normal behavior. The MEG data obtained from epileptic areas had higher amplitudes than the MEG from normal regions and were comparable with the theoretical magnetic fields from Poisson and Gauss distributions.

Anninos et al. (28) provided information from neural models on the role played by the specific anatomy of various brain centers in determining their function. The experimental approach proposed here involved a comparison between MEG in normal and epileptic patients and an analysis of model nerve net using computer stimulation in a large general-purpose digital computer.

\section{Neonatal MEG}

Kotini et al. (29) investigated the presence of any non-linearity in the MEG recordings in neonates born to preeclamptic pregnancies in comparison with the ones born to uncomplicated pregnancies, in order to find out the differences in the mechanisms underlying their brain waves. The above analysis of the MEG in neonatal preeclamptic brain exhibited a lower dimension complexity compared to the normal neonatal brain, as well as the first Lyapunov exponent, which mean lower information processing.

Anastasiadis et al. (30) performed a prospective study of neonatal brain function on respective term neonates who were delivered normally, without any clinical signs of brain damage. These MEGs were low in almost all neonates from the "normal pregnancy" group, while they were high in most neonates from the "pre-eclamptic" group. The difference between the two groups was statistically significant. A statistically significant difference in spectral amplitudes of neonatal brain activity was observed between normal term and pre-eclamptic neonates.

\section{Shizophrenia}

Kotini et al. (31) investigated the presence of any non-linearity in the MEG from the temporal lobe of schizophrenic patients in comparison with controls, in order to find the differences underlying the brain waves. There were no significant differences between the two groups as far as age and sex were concerned. The analysis of the MEG in the schizophrenic group exhibited lower dimension complexity. Moreover the first Lyapunov exponent showed lower values compared with the corresponding ones in the control group, which mean lower information processing.

\section{Alzheimer}

Anninos et al. (32) obtained MEG from the brain of patients suffering from AD. Furthermore, chaotic dynamic methods were used, in order to estimate the correlation dimension D of the reconstructed phase spaces. The estimated values of $\mathrm{D}$, in conjunction with the results derived from the order data analysis methods, strongly supported the existence of low dimension chaotic structures in the dynamics of cortical activity of $\mathrm{AD}$ patients.

\section{The TMS electronic device}

The TMS electronic device is a modified helmet containing up to 122 coils which are arranged in five array groups, so as to cover the main 5 brain regions (frontal, vertex, right and left temporal and occipital regions) of the subject. It is designed to create TMS range modulations of magnetic flux in the alpha frequency range $(8-13 \mathrm{~Hz})$ of each patient. The TMS device was configured for each individual to generate a square wave (so as to resemble the firing activity of neurons in the brain) modulated magnetic field at the individual's mean peak alpha frequency $(33,34)$.

\section{Discussion}

The ionic currents which originate from biochemical sources at the cellular level in the CNS produce electric and magnetic fields. The generation of EEG signals in the brain, is an exact way to determine the potential distribution at the scalp given a set of intracerebral current sources. EEG can only be measured at a considerable distance from the source if the responsible neurons are regularly arranged and activated in a more or less synchronous way. Thus, while with the EEG it is very difficult to localize where a particular signal originates in the brain, in the case of the magnetic field it is easier to localize the signals origin in the brain. The MEG is presently regarded as the most efficient method for recording the brain activity in real time for many reasons.

Compared with the EEG, the MEG has unique sensitivity to the CNS disorders and normal functions of the brain. In addition, the MEG offers functional mapping information and measurement of brain activity in real time, unlike CT, MRI and fMRI which only provide structural, anatomical and metabolic information. With the MEG the brain is seen in 'action' rather than viewed as a still image. Another most important point is that the MEG has far more superior ability to resolve millisecond temporal activity associated with the processing of information which is the main task of our brain. Other properties of the MEG that should be mentioned are the following: Neither electrodes nor a reference point are necessary for recording the MEG compared to the EEG; the transducers for the MEG need not touch the scalp, because the magnetic field does not disappear where conductivity is zero. The recordings of the MEG are the measurements of the magnetic fields perpendicular to the skull, which are caused by tangential current sources. By contrast, the EEG is a measure of both components. This means MEG measures the cortical activity lying in the sulci and not in the convexity of the gyri (35-37). From the measured field, it is possible, by making appropriate assumptions, to calculate backwards 
the activated brain area. In interpreting MEG data, one is dealing with the electromagnetic inverse problem, i.e., with the calculation of the source currents responsible for the measured extracranial magnetic field with the use of ECD. Matching an ECD model to the extracranial magnetic field became a simple and convenient method for localizing sources of cerebral activity. Experimental studies proved the accuracy of ECD localization of sources of interictal discharges (38-44).

The most important clinical results derived by the use of MEG are obtained from the preoperative examination of sources of epileptic activity and the surgical planning in patients with brain tumors. The knowledge of the position of the primary sensory areas and those involved in speech production and comprehension is a prerequisite for avoiding potential neurological deficits during the surgery (45-48). Source localization of MEG spikes is often performed in clinical practice for identifying an irritative zone. The source analysis of MEG signals incorporates anatomical information derived from the MRI with the sources of neural activities from each patient providing a current dipole distribution map. The ECD analysis has been widely used for source localization of epileptic spikes for decades. It assumes that a single dipole source generates all the neuromagnetic fields recorded from the sensors. Adequate ECDs are mapped on the patient's MRI (45, 46). It is a well established procedure with some limitations. The inverse problem localizes in three dimensional space the neural sources of the MEG recordings. Methods to solve the inverse problem need to make additional assumptions according to the experimental protocol. An alternative method to overcome the inverse problem is to use the beamforming (49). This method extracts the origins of a signal from a specified spatial location. MEG investigates also oscillatory brain activity by using Fourier, Wavelet or Hilbret transformations showing significant changes in the power and synchronization of the oscillatory activity (50).

The MEG spike morphology has not been adequately described while the EEG spikes were well defined. A clinical guideline has recommended indentifying the MEG spikes based on the principles established for EEG (51). The MEG spikes are useful for clinical application mostly in patients with negative EEG (46).

Consequently, the MEG could provide clinical practice with a non-invasive and rapid method, which could be adjunct to conventional methods for the assessment of brain dysfunction.

\section{References}

1. Gemousakakis T, Anninos P, Zissimopoulos A, et al. A study on the age dependency of gustatory states: Low-frequency spectral component in the resting-state MEG. J Integr Neurosci 2013; 12: 1-13.

2. Gemousakakis T, Kotini A, Anninos P, Zissimopoulos A, Prassopoulos P. MEG evaluation of taste by gender difference. J Integr Neurosci 2011; 10: 537-45.
3. Anninos P, Kotini A, Adamopoulos A, et al. Identification of taste quality with the use of MEG. J Integr Neurosci 2006; 5: 535-540.

4. Antoniou P, Adamopoulos A, Anninos PA, Piperidou H, Kotini, A. Assessing brain pathophysiology through non linear analysis of MEG in Idiopathic Generalized Epilepsy cases. J Behav Brain Sci 2012; 2: 445-453.

5. Kotini A, Mavraki E, Anninos P, Piperidou H, Prassopoulos P. Magnetoencephalographic findings in two cases of juvenile myoclonus epilepsy. Brain Topogr 2010; 23: 41-5.

6. Anninos P, Kotini A, Dipla V, Tsalkidis A, Chatsimichail A. MEG evaluation of Febrile Seizures in Young Children. J Child Neurol 2010; 25: 61-6.

7. Kotini A, Mavraki E, Anninos P, Piperidou H, Prassopoulos P. MEG evaluation of epileptic activity in the time and frequency domain. J Integr Neurosci 2008; 7: 463-80.

8. Abatzoglou I, Anninos P, Tsalafoutas I, Koukourakis M. Multi-channel magnetoencephalogram on Alzheimer disease patients. J Integr Neurosci 2009; 8: 13-22.

9. Anninos P, Kotini A, Anninou N, Adamopoulos, A, Papastergiou, A, Tsagas, N. MEG recordings of patients with CNS disorders before and after external magnetic stimulation. J Integr Neurosci 2008; 7: 17-27.

10. Anninos P, Adamopoulos A, Kotini A, Tsagas N, Tamiolakis D, Prassopoulos P. MEG evaluation of Parkinson's diseased patients after external magnetic stimulation. Acta Neurol Belg 2007; 107: 5-10.

11. Kotini A, Anninos P, Adamopoulos A, Prassopoulos P. Low Frequency MEG Activity and MRI Evaluation in Parkinson's Disease. Brain Topogr 2005; 18: 59-63.

12. Antoniou P, Anninos P, Piperidou H, et al. Treatment evaluation of brain tumors using non-linear analysis of Magnetoencephalographic data. A novel technique. Gazz Med Ital 2004; 163: 285-290.

13. Antoniou P, Anninos P, Piperidou H, et al. Non linear analysis of Magnetoencephalographic signals as a tool for assessing malignant lesions of the brain; first results. Brain Topogr 2004; 17: 117-123.

14. Abatzoglou I, Anninos P, Adamopoulos A, Koukourakis M. Nonlinear analysis of brain magnetoencephalographic activity in Alzheimer disease patients. Acta Neurol Belg 2007; 107: 34-9.

15. Kotini A, Anninos P, Tamiolakis D. MEG mapping in multiple sclerosis patients. Eura Medicophys 2007; 43: 345-8.

16. Kotini A, Anninos P, Tamiolakis D, Prassopoulos P. Differentiation of MEG activity in multiple sclerosis patients with the use of nonlinear analysis. J Integr Neurosci 2007; 6: 233-40.

17. Anninos P, Kotini A, Tamiolakis D, Prassopoulos P. Evaluation of an intracranial arachnoid cyst with MEG after external magnetic stimulation. J Integr Neurosci 2007; 6: 227-32.

18. Anninos P, Kotini A, Tamiolakis D, Tsagas N. Transcranial magnetic stimulation. A case report and review of the literature. Acta Neurol Belg 2006; 106: 26-30.

19. Anninos P, Karpouzis A, Kotini A, Kouskoukis C. Exogenous magnetic stimulation in therapeutic management of universalis alopecia areata. Gazz Med Ital 2004; 163: 281-284.

20. Anninos P, Kotini A, Adamopoulos A, Tsagas N. Magnetic stimulation can modulate seizures in epileptic patients. Brain Topogr 2003; 16: 57-64.

21. Anninos P, Tsagas N, Jacobson J, Kotini A. The biological effects of magnetic stimulation in epileptic patients. Panminerva Med 1999; 41: 207-15.

22. Anninos P, Tsagas N, Jacobson J, Kotini A. The biological effects of magnetic stimulation in epileptic patients. Review Series Neurology 2001; 2: 15-17.

23. Anninos P, Kotini A, Adamopoulos A, Tsagas N. The use of nonlinear analysis for differentiating brain biomagnetic activity in epileptic patients before and after magnetic stimulation. Hardronic J Suppl 1999; 14: 1-26.

24. Anninos P, Adamopoulos A, Kotini A, Tsagas N. Nonlinear Analysis of Brain Activity in Magnetic Influenced Parkinson Patients. Brain Topogr 2000; 13: 135-144. 
25. Anninos P, Tsagas N, Kotini A, Adamopoulos A. The chaos theory for differentiating brain biomagnetic activity in normal and multiple sclerosis patients before and after magnetic stimulation. Hardronic J Suppl 1999; 14: 137-151.

26. Anninos P, Tsagas N, Kotini A, Konstantinopoulos, P. The use of nonlinear analysis for differentiating brain biomagnetic activity in normal and Meniere's syndrome patients before and after magnetic stimulation. Hardronic J Suppl 1999; 14: $153-170$.

27. Kotini A, Anninos P, Anastasiadis AN, Tamiolakis D. A Comparative Study of a Theoretical Neural Net Model with MEG Data from Epileptic Patients and Normal Individuals. Theor Biol Med Model 2005; 2: 37.

28. Anninos P, Kotini A, Papastergiou A, Tsagas N. Neural Modeling approach to determine Structure and Function in Brain Center. Hardronic J Suppl 2000; 15: 154-184.

29. Kotini A, Koutlaki N,Anninos P, Adamopoulos A, Liberis V, Anastasiadis P. Chaotic analysis approach in neonatal MEG. Biol Neonate 2003; 84: 214-221.

30. Anastasiadis P, Anninos P, Koutlaki N, Kotini A, Avgidou K, Adamopoulos A Neonatal MEG and spectral analysis. Clin Exp Obstet Gynecol 2001; 28: 269-273.

31. Kotini A, Anninos P. Detection of non-linearity in schizophrenic patients using MEG. Brain Topogr 2002; 15: 107-113.

32. Anninos P, Kotini A, Adamopoulos A, Tsagas N, Jacobson J. Nonlinear Analysis of MEG Activity Recorded in Alzheimer Patients. Hardronic J Suppl 2000; 15: 1-16.

33. Anninos PA, Tsagas N, Sandyk R, Derpapas K (1991) Magnetic stimulation in the treatment of partial seizures. Int J Neurosci 60: 141-171.

34. Anninos PA, Tsagas N (1995) Electronic apparatus for treating epileptic individuals. US patent 5453072 Sept 26

35. Bast T, Wright T, Boor R, et al. Combined EEG and MEG analysis of early somatosensory evoked activity in children and adolescents with focal epilepsies. Clin Neurophysiol 2007; 118: 1721-35.

36. Ramantani G, Boor R, Paetau R, et al. MEG versus EEG: influence of background activity on interictal spike detection. J Clin Neurophysiol 2006; 23: 498-508.

37. Rodin E, Funke M, Berg P, Matsuo F. Magnetoencephalographic spikes not detected by conventional electroencephalography. Clin Neurophysiol 2004; 115: 2041-7.

38. Hamalainen M, Hari R, Ilmoniemi R, Knuutila J, Lounasmaa OV. Magnetoencephalography: theory, instrumentation, and applications to non-invasive studies of the working human brain. Rev Mod Phys 1993; 65: 413-497.
39. Ishii R, Canuet L, Aoki Y, et al. Frequency diversity of posterior oscillatory activity in human revealed by spatial filtered MEG. J Integr Neurosci 2013; 12: 343-53.

40. Iwaki S, Bonmassar G, Belliveau JW. Dynamic cortical activity during the perception of three-dimensional object shape from two-dimensional random-dot motion. J Integr Neurosci 2013; 12: 355-67.

41. Kishida K. Neurodynamics of somatosensory cortices studied by magnetoencephelography. J Integr Neurosci 2013; 12: 299-329.

42. Knowlton RC, Laxer KD, Aminoff MJ, Roberts TP, Wong ST, Rowley HA. Magnetoencephalography in partial epilepsy: clinical yield and localization accuracy. Ann Neurol 1997; 42: 622-631.

43. Oishi M, Kameyama S, Masuda H, et al. Single and multiple clusters of magnetoencephalographic dipoles in neocortical epilepsy: significance in characterizing the epileptogenic zone. Epilepsia 2006; 47: 355-364.

44. Tonoike M, Yoshida T, Sakuma H, Wang LQ. fMRI measurement of the integrative effects of visual and chemical senses stimuli in humans. J Integr Neurosci 2013; 12: $369-84$.

45. Stufflebeam S. Clinical magnetoencephalography for neurosurgery. Neurosurg Clin N Am 2011; 22: 153-67.

46. Tanaka N, Stufflebeam S. Clinical application of spatiotemporal distributed source analysis in presurgical evaluation of epilepsy. Front Hum Neurosci 2014; 8: 62.

47. Dorfer C1, Widjaja E, Ochi A, Carter Snead Iii O, Rutka JT. Epilepsy surgery: recent advances in brain mapping, neuroimaging and surgical procedures. J Neurosurg Sci 2015 Jun; 59(2): 141-55.

48. Pizzella V, Marzetti L, Della Penna S, de Pasquale F, Zappasodi F, Romani GL. Magnetoencephalography in the study of brain dynamics. Funct Neurol 2014 Oct-Dec; 29(4): 241-53.

49. Woolrich M, Hunt L, Groves A, Barnes G. MEG beaforming using Bayesian PCA for adaptive data covariance matrix regularization. Neuroimage 2011; 57: 1466-79.

50. Proudfoot M, Woolrich MW, Nobre AC, Turner MR. Magnetoencephalography. Pract Neurol 2014 Oct; 14(5): 336-43.

51. Bagic, AI, Knowlton RC, Rose DF, Ebersole JS, ACMEGS Clinical Practice Guideline (CPG) Committee (2011). American clinical magnetoencephalography society clinical practice guideline 1 : recording and analysis of spontaneous cerebral activity. J Clin Neurophysiol 2011; 28, 348-354.

Received: 26/03/2015

Accepted in revised form: 30/07/2015

\section{Corresponding author:}

Associate Professor A. Kotini, Lab of Medical Physics, Medical School, Democritus, University of Thrace, University Campus, 68100, Alexandroupolis, Greece; e-mail: akotini@med.duth.gr 